\title{
Erratum: Calculated electron dynamics in an electric field [Phys. Rev. A 56, 278 (1997)]
}

\author{
F. Robicheaux and J. Shaw \\ (Received 8 July 2016; published 10 August 2016)
}

DOI: 10.1103/PhysRevA.94.029904

In our work the statement above Eq. (21) defines the matrix $\mathcal{S}$ as a nonunitary $S$ matrix. The characterization of this matrix as nonunitary is wrong. This matrix is unitary as shown in Sec. IIIA of Ref. [1]. This misclassification of the matrix $\mathcal{S}$ does not affect the correctness of any of the equations or results of our work. The unitarity of the matrix $\mathcal{S}$ means the overlap matrix in Eq. (24) of our work is the unit matrix. Thus, the subsequent formulas with the overlap matrix can be simplified.

In the event that someone uses the expressions for the dipole matrix element in the case of a truly nonunitary $S$ matrix, the role of the dipole defined in Eq. (25) is not clearly specified. By defining this dipole as $D_{\beta}^{-}(E)=\left(O^{-1} D^{-}\right)_{\beta}$, one uses it to obtain the time-dependent wave function through $\Psi(t)=\int d E \sum_{\beta} D_{\beta}^{-}(E) \Psi_{E \beta m}^{-} A(E)$ where the $A(E)$ is the amplitude of the energy component of the laser relative to the ground state. The cross section can be obtained by noting that $\sigma \propto \sum_{\beta \beta^{\prime}} D_{\beta}^{-*}<\Psi_{\beta} \mid \Psi_{\beta^{\prime}}>$ $D_{\beta^{\prime}}^{-} \propto \sum_{\beta \beta^{\prime}} D_{\beta}^{-*} O_{\beta \beta^{\prime}} D_{\beta^{\prime}}^{-}$.

We acknowledge L. Zhao for pointing out the importance of the statement in our work that the $S$ matrix was nonunitary, which led to the derivation that it was, in fact, unitary.

[1] P. Giannakeas, F. Robicheaux, and C. H. Greene, Phys. Rev. A 91, 043424 (2015). 